\title{
Capacitance DNA bio-chips improved by new probe immobilization strategies
}

\author{
Sandro Carrara ${ }^{\mathrm{a}, *}$, Andrea Cavallini ${ }^{\mathrm{a}}$, Yusuf Leblebici ${ }^{\mathrm{a}}$, Giovanni De Micheli ${ }^{\mathrm{a}}$, Vijayender Bhalla ${ }^{\mathrm{b}}$, \\ Francesco Valle ${ }^{b, 1}$, Bruno Samorì ${ }^{b}$, Luca Benini ${ }^{c}$, Bruno Riccò ${ }^{c}$, Inger Vikholm-Lundin ${ }^{d}$, Tony Munter ${ }^{d}$ \\ a EPFL, Swiss Federal Institute of Technology, Lausanne CH-1015 Lausanne (CH), Switzerland \\ ${ }^{\mathrm{b}}$ Biochemistry Department, Bologna University (IT), Bologna \\ ${ }^{\mathrm{c}}$ D.E.I.S. Department, Bologna University (IT), Bologna \\ ${ }^{\mathrm{d}}$ Technical Research Centre of Finland (VTT), Tampere, Finland
}

\section{A R T I C L E I N F O}

\section{Article history:}

Received 21 September 2009

Accepted 18 January 2010

Available online 25 February 2010

\section{Keywords:}

Biochip

DNA immobilization

Ethylene-glycol

Alkanethiols

Mercapto-hexanol

Lipoate-diethanolamines

\begin{abstract}
A B S T R A C T
Label-free DNA detection plays a crucial role in developing point-of-care biochips. Capacitance detection is a promising technology for label-free detection. However, data published in literature often show evident time drift, large standard deviation, scattered data points, and poor reproducibility. To address these problems, mercapto-hexanol or similar alkanethiols are usually considered as blocking agents. The aim of the present paper is to investigate new blocking agents to further improve DNA probe surfaces. Data from AFM, SPR, florescence microscopy, and capacitance measurements are used to investigate new lipoate and ethylene-glycol molecules. The new surfaces offer further improvements in terms of diminished detection errors. Film structures are investigated at the nano-scale to justify the detection improvements in terms of probe surface quality. This study demonstrates the superiority of lipoate and ethylene-glycol molecules as blocking candidates when immobilizing molecular probes onto spot surfaces in label-free DNA biochip.
\end{abstract}

(c) 2010 Elsevier Ltd. All rights reserved.

\section{Introduction}

Medical diagnosis requires point-of-care biosensor arrays at the patient's bed. This requirement is due to new emerging demands for personalized therapies because therapeutic agents amount in tissue and blood serum is different on a patient-bypatient basis [1]. Therefore, the development of low-cost, pointof-care technologies for array biochips is a necessary step to introduce personalized therapies in clinical practice. The usually considered micro-array technology based on optical detection and molecular labeling is costly and time consuming. Thus, it is not adapted for applications to personalized therapy in hospital or at home. Label-free capacitance DNA biochips are a valid solution as they present many advantages. After the initial works of Mirsky $[2,3]$, the application of capacitance detection for DNA [4], interleukin [5] and heavy metals [6] was extensively investigated in late 90 s. After 2003, the capacitance detection was pursued by increased vigor as demonstrated by more recent works published by different research groups. It was showed that this detection principle can be applied to DNA hybridization by immobilizing

\footnotetext{
* Corresponding author.

E-mail address: sandro.carrara@epfl.ch (S. Carrara).

${ }^{1}$ Present address: ISMN-CNR, Bologna.
}

single strand probe DNA molecules onto gold [7] and silicon [8], as well as PNA probe molecule [9]. Capacitive method has been used to reach femtomolar concentration range in metal ions detection [10], to check antibody affinity on macroporus silicon [11] and gold [12]. Finally, the possibility to develop fully integrated DNA biochip was demonstrated $[13,14]$. Good reviews about this large effort were published summarizing applications for pathogenic detection [15], and mechanisms of impedance change [16]. However, the usually considered probes immobilization does not present enough stable capacitance properties. Evident time drift [2,13], large standard deviation [14], scattered data points [4], and poor reproducibility [5] usually affect the detection signals. All these phenomena are related to electrode/solution interfaces which are not a perfect insulator [17]. Thus, the sensing capability is reduced. Special efforts are dedicated word wide to probes surface improvement in biosensors. New materials based on lipoamide (the lipa-DEA [18,19]) and on ethylene-glycol $[20,21]$ were applied to improve surface plasmon resonance (SPR) biosensors. A large decrease of non-specific adsorption on probe surfaces was demonstrated for both lipoates [18] and ethylene-glycol alkanethiols [21]. The aim of this paper is to demonstrate that these new probe functionalizations assure an improved stability in DNA detection by using capacitive biochips, too. The paper presents original investigations on lipa-DEA and ethylene-glycol monolayers. The study was conducted by 
comparing with performances obtained with the usually considered mercapto-hexanol. Results from AFM, fluorescence, SPR and capacitance measurements on chip are used to demonstrate that these new functionalizations are innovative and suitable to improve label-free DNA biochip detectors.

\section{Materials and methods}

\subsection{Biochip fabrication}

A standard lift-off process is used to pattern the gold electrodes onto glass substrates. To improve adhesion between substrate and electrodes, first a $20 \mathrm{~nm}$ layer of chromium is deposited, followed by a $200 \mathrm{~nm}$ layer of Gold using thermal evaporation. The entire surface is covered by a thick $(10 \mu \mathrm{m})$ layer of AZ1512 photoresist as passivation layer. Individual sensor spots are exposed by developing AZ1512. The chip contains a total of 32 square electrode arranged in groups of four electrode pairs. The electrode square side is $200 \mu \mathrm{m}$ and the electrode's separation is $20 \mu \mathrm{m}$.

\subsection{Capacitance measurements}

Charge-based capacitance measurement ( $\mathrm{CBCM}$ ) technique is employed to improve capacitance estimation. With this method, capacitance values are estimated by measuring the current transient due to RC behavior of the electrodes/solution biointerface driven by a square bias voltage. This bias is used to drive the equivalent capacitance between working and reference electrodes of each sensing area of the biochip. As shown in Fig. 1, the current at the electrochemical bio-interface may be written as

$i(t)=I_{d c}+i_{C}(t)$

by accounting for some leakages passing through the biomolecular layer. The average charging-current and the interface capacitance are then related by [14]:

$I_{A V G}=\frac{I_{d c}}{2}+\frac{1}{T} \int_{0}^{T / 2} i_{C}(t) d t=\frac{I_{d c}}{2}+C V_{\text {Step }} f$.

This equation shows a direct relationship between the average current of square driving signal and its frequency. The slope of this linear relationship returns the bio-interface capacitance. Fig. 1 also shows a possible CMOS implementation of this approach. In the design, a pulse generator is used to drive four switches required to generate the squared bias. The current emerging from the bio-interface drives other four switches to rectify the current transients which are used as input of an integrator. The integrator output is directly related to the current average flowing through the bio-interface. A sweep in frequency and the subsequent acquisitions of different average currents enable the estimation of the bio-interface equivalent capacitance by means of a simple computational algorithm. A VLSI biochip implementing the CMOS architecture reported in Fig. 1 has been realized [14]. However, a precise estimation of the equivalent bio-interface capacitance from Eq. (2) is possible only if that capacitance is not varying with the frequency. Unfortunately, it has already been demonstrated that the interface equivalent capacitance is varying in frequency both on bare gold electrodes [14] and on DNA probes directly immobilized onto gold electrodes [17].

Therefore, an alternative method for precise capacitance estimations has been proposed to overcome this problem. The frequency-to-capacitance measurement (FTCM) technique [13] is based on the relation between RC parameter and charging time at the bio-interface. In this case, a current generator charges the equivalent capacitance and the potential of that charging is used as input of a comparator, as shown in Fig. 2(A). Once the comparator threshold is overcome, then the comparator output switches up and the input polarity at the capacitance is reversed as well as that of the threshold generator. Thus, the bio-interface discharges towards negative potentials till the reversed threshold is reached, as shown in Fig. 2(B). Considering the exponential shape of the charging curves, the period of comparator switching may be related to threshold by the equation [13]:

$T=2 R C \ln \left(\frac{1}{1-\left(\left(V_{\text {ref }}\right) /\left(I_{\text {ref }} R\right)\right)}\right)$.

By adjusting current and voltage references, an approximated relation between the frequency of comparator switching and the capacitance of bio-interface is then possible:

$f=I_{\text {ref }}\left(2 V_{\text {ref }} C\right)^{-1}$.

Therefore, an easy and precise measure of the frequency by means of usual counters returns a precise estimation of the biointerface equivalent capacitance. Although this method avoids the problem of frequency-dependant capacitances, unfortunately it cannot address the problem of time-trends in frequency signals if the bio-interfaces are not enough stable [13]. The problem of biointerfaces not enough stable-in-time may be addressed by developing special DNA probe immobilizations. In our laboratory, both the above mentioned methods for precise estimation of biointerfaces capacitance have been tested by considering different kinds of DNA probe immobilization.

\subsection{Bio-interface chemicals}

Ethylene-glycol functionalized alkanethiols differently terminated $\left(\mathrm{SH}-\left(\mathrm{CH}_{2}\right)_{11}-\left(\mathrm{OCH}_{2} \mathrm{CH}_{2}\right)_{3} \mathrm{OCH}_{2} \mathrm{COOH}\right.$, and $\mathrm{SH}-\left(\mathrm{CH}_{2}\right)_{11}-$ $\left.\left(\mathrm{OCH}_{2} \mathrm{CH}_{2}\right)_{3} \mathrm{OH}\right)$ were purchased from Prochimia, Poland. 11-Mercaptoundecanoic acid (HS- $\left.\left(\mathrm{CH}_{2}\right)_{10} \mathrm{COOH}\right)$, 6-mercapto-1-

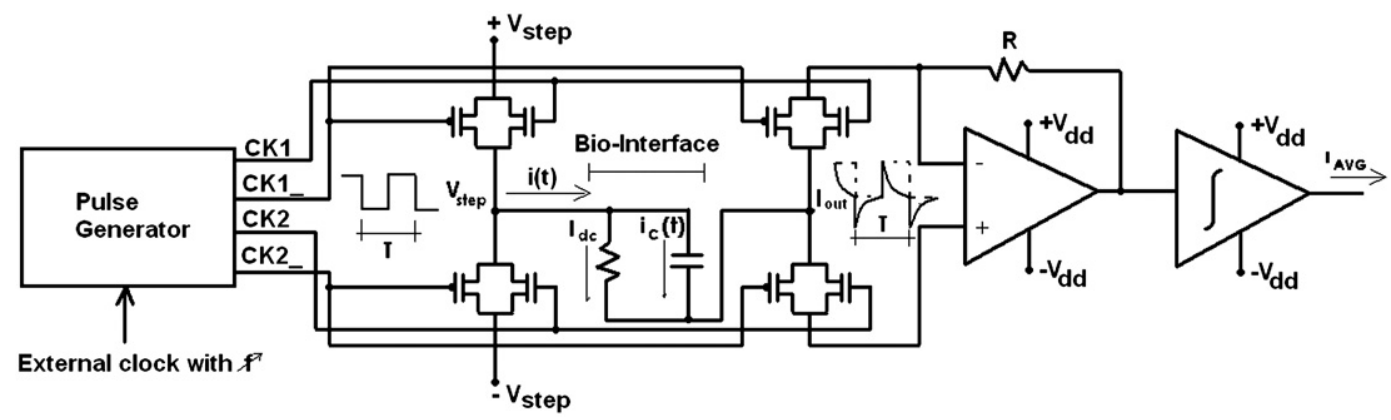

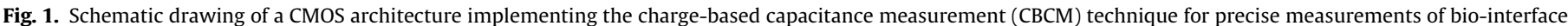
capacitance. 
A

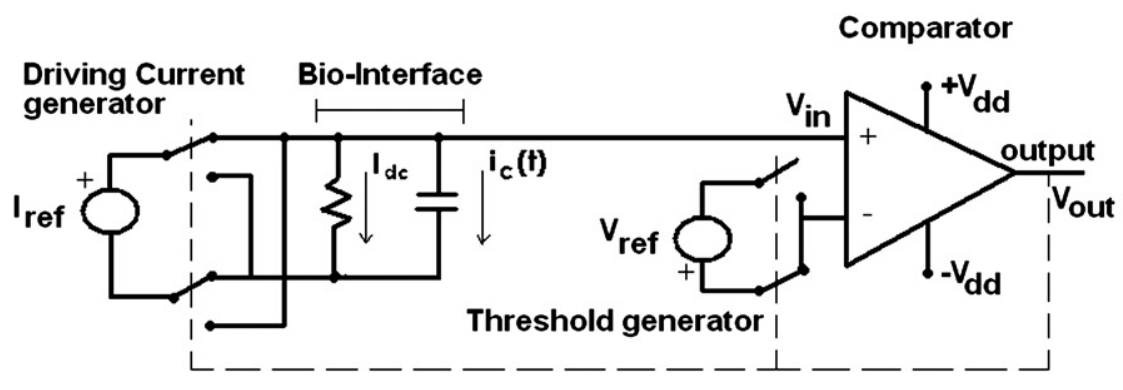

B

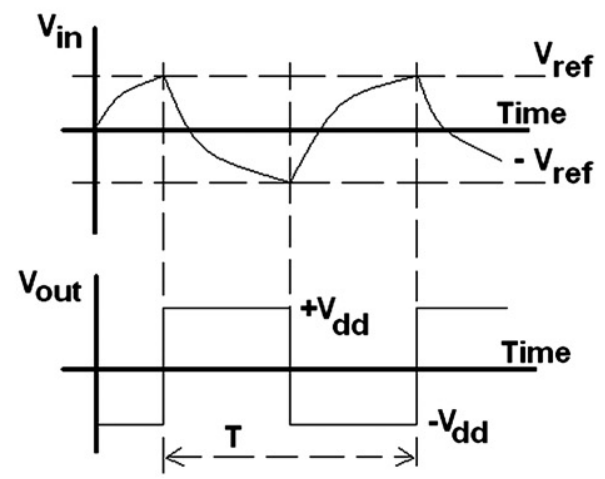

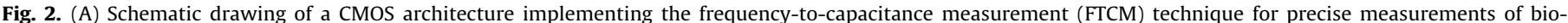
interface capacitance. (B) Time signals related to input and output of the comparator.

A

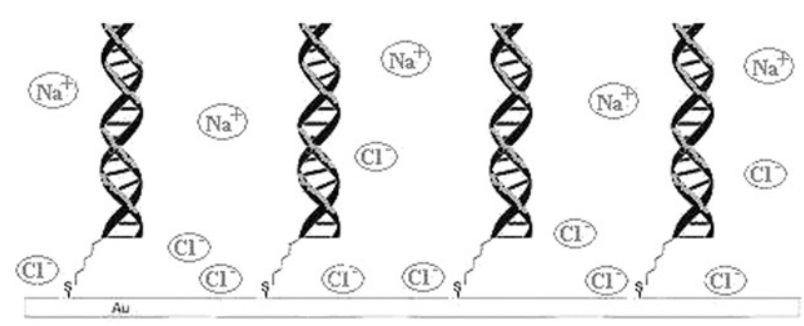

C
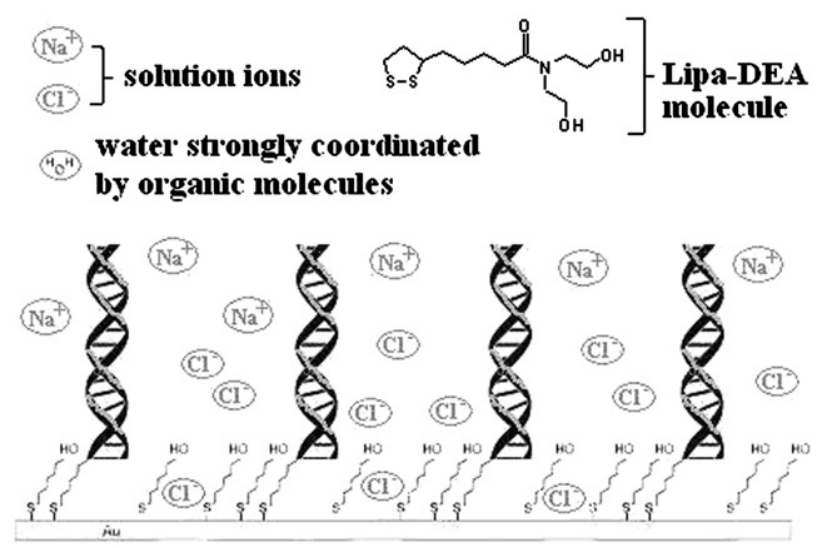

B

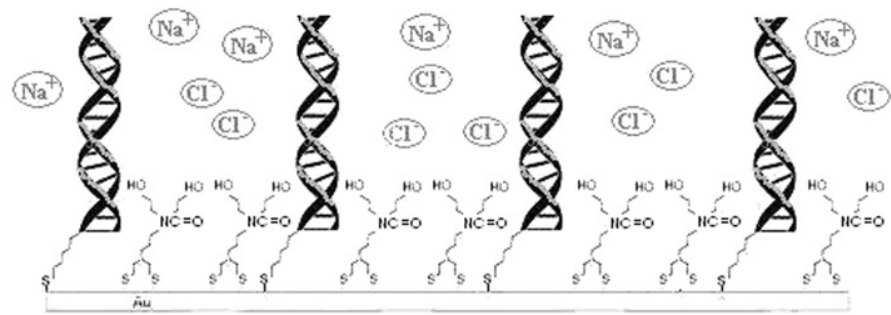

D

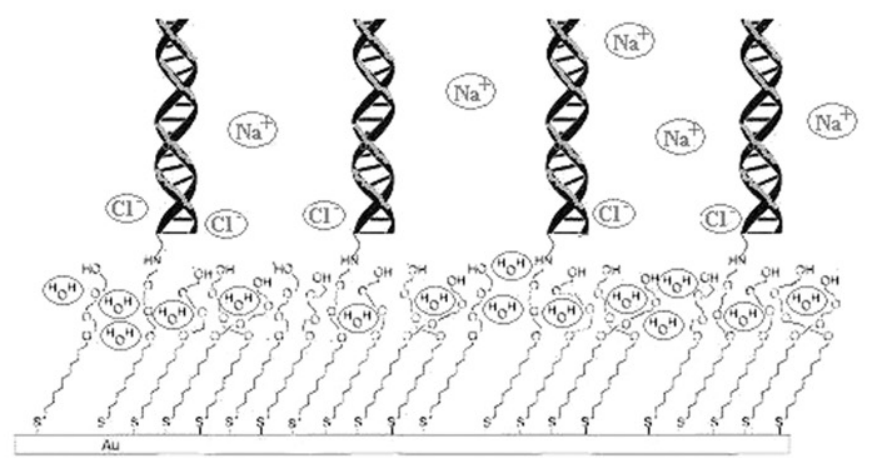

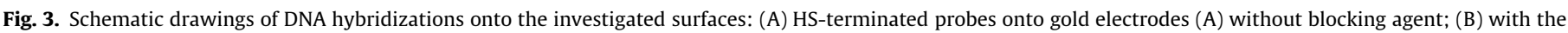
usually considered 6-mercapto-1-hexanol or (C) with Lipa-DEA; (D) NH-terminated probes onto ethylene-glycol precursors.

hexanol, $\mathrm{NaCl}, \mathrm{Na}_{2} \mathrm{HPO}_{4}, \mathrm{KH}_{2} \mathrm{PO}_{4}, \mathrm{KCl}, \mathrm{H}_{2} \mathrm{O}_{2}$ (50\%) and absolute ethanol were purchased by Sigma, Switzerland. Different singlestranded DNA (ssDNA) probe molecules of the same length (25-mer), thiol or amino modified, with spacers presenting chains of six carbon atoms were supplied by MWG Biotech, Germany. Single-stranded DNA target were acquired by MWG as well. $N, N$ bis(2-hydroxyethyl)- $\alpha$-lipoamide (Lipa-DEA) was prepared according to a published method [22]. All the chemicals were used without further purification.

\subsection{DNA probes immobilization}

Different kinds of probe immobilizations were prepared in order to check different sensing monolayers. Mainly, three different immobilization techniques were tested, as shown in
Fig. 3: SH-terminated ssDNA are immobilized directly onto chip gold electrodes (Fig. 3(A)) and, then, 6-mercapto-1-hexanol is added as blocking agent (Fig. 3(B)) following a well established procedure [4]. SH-terminated ssDNA are mixed to Lipa-DEA molecules and co-adsorbed onto the chip gold electrodes (Fig. 3(C)). The co-immobilization of probes and blocking molecules was found to be more efficient than that with the DNA probes surface pre-formed and then post-treated with LipaDEA [19]. Ethylene-glycol monolayers are formed as probe precursors onto the electrode surface (Fig. 3(D)). The layers are obtained from a mixture of two differently modified alkanethiol molecules ( $\mathrm{HS}-\left(\mathrm{CH}_{2}\right)_{11}\left(\mathrm{OCH}_{2} \mathrm{CH}_{2}\right)_{3} \mathrm{OH}$ and $\mathrm{HS}-\left(\mathrm{CH}_{2}\right)_{11}$ $\left.\left(\mathrm{O}-\mathrm{CH}_{2} \mathrm{CH}_{2}\right)_{3} \mathrm{OCH}_{2} \mathrm{COOH}\right)$. The mixture is prepared into a $2 \mathrm{mM}$ final concentration solution of ethanol with proportion $1.96 \mathrm{mM}$ of $\mathrm{OH}$-terminated thiols and $0.04 \mathrm{mM}$ of $\mathrm{COOH}$-terminated thiols. The samples were incubated overnight, under dark conditions, in 
A

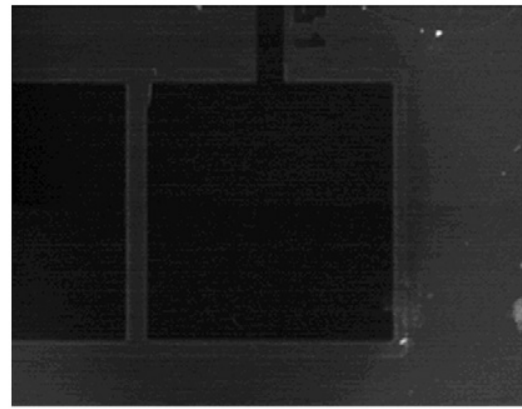

B

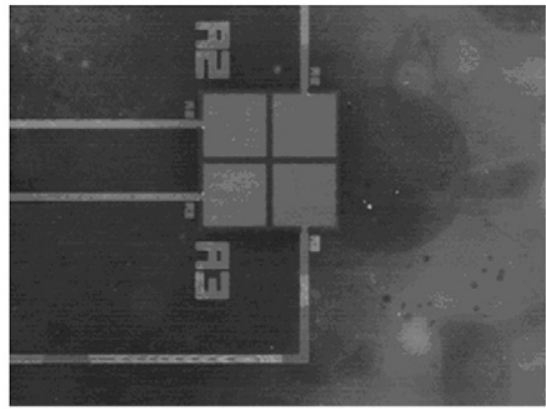

Fig. 4. Optical microscopy images of chip spots without (A) and with (B) ssDNA probes marked with fluorescence and immobilization onto gold electrodes.

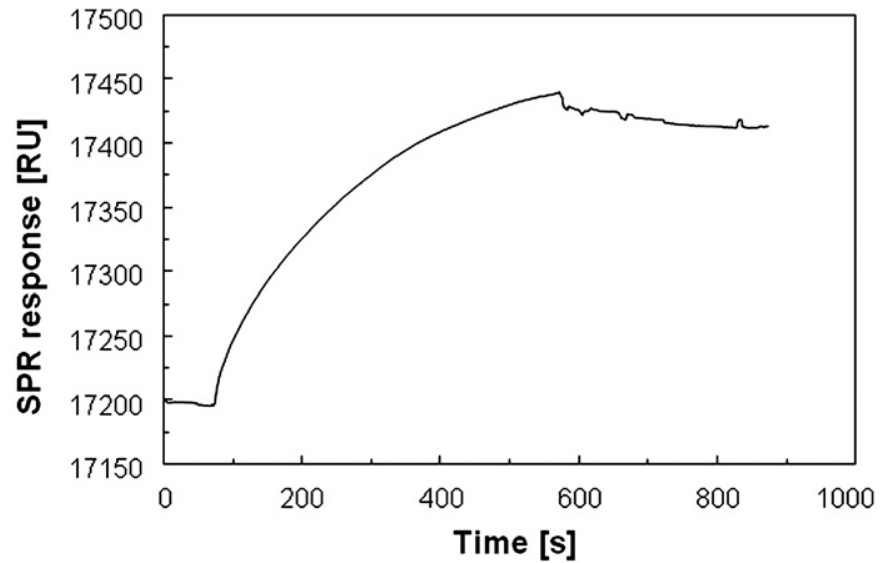

Fig. 5. Hybridization of a $60 \mu \mathrm{g} / \mathrm{ml}$ SH-oligo/Lipa-DEA monolayer with $0.5 \mu \mathrm{g} / \mathrm{ml}$ complementary DNA investigated by SPR.

such a mixture. The samples were then rinsed and sonicated in ethanol for $10 \mathrm{~min}$. Finally, NH-terminated ssDNA probes are anchored to the precursor monolayers by using well known procedures based on $\mathrm{N}$-hydroxysuccinimide (NHS) and of $N$-Ethyl- $N$-(3-dimethylaminopropyl) carbodiimide (EDC) [21]. After the SAM formation and before the capacitance measurements, the biochips were left for conditioning in PBS buffer, in dark, for $24 \mathrm{~h}$. The conditioning was necessary to further stabilize the capacitance measurements on the so prepared electrode chips.

By following the same procedures, DNA probe films were also immobilized onto template stripped gold (TSG) and onto Biacore gold chips for AFM and SPR investigations, respectively. The different functionalizations were verified by using fluorescence microscopy and SPR, as shown in Figs. 4 and 5.

\subsection{AFM measurements}

Atomic force microscopy (AFM) imaging was performed in tapping mode with PointProbe nanocontact silicon probes on a Nanoscope IIIa SFM system equipped with a multimode head and a type A piezoelectric scanner (Veeco, Santa Barbara, CA, USA). The images were acquired in ethanol by using a 'liquid cell'. Raw images have been processed only for background removal (flattening) by using the microscope manufacturer's image processing software package. Line profiles have been obtained by using ImageJ ver. 1.410 . This software is provided for free by Wayne Rasband, from National Institute of Health, US.

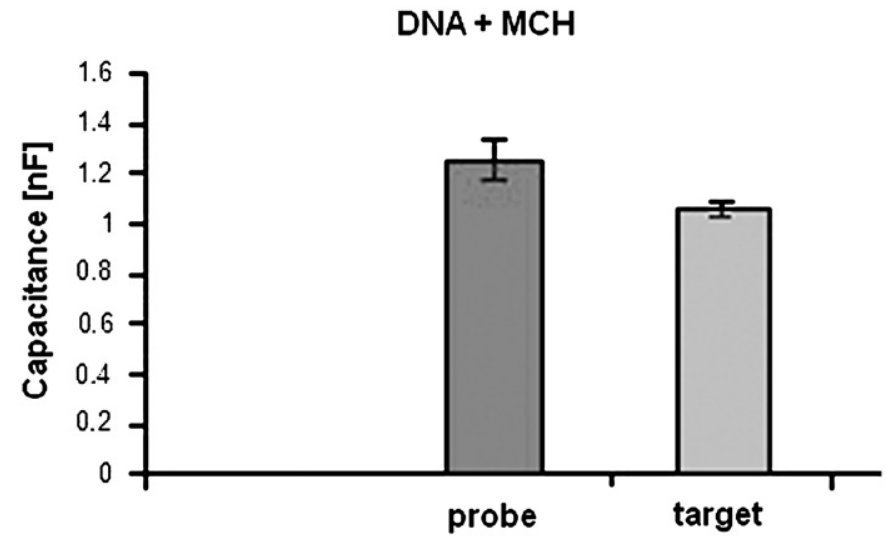

Fig. 6. DNA detection by using 6-mercapto-1-hexanol as blocking agent.

\section{Results}

Results of DNA hybridization detection by using the different proposed DNA probe immobilizations are summarized in this section. In particular, data are presented in terms of average capacitance signals and their standard deviations in 10-min series of acquisition on the same chip spot. AFM images and profiles are presented in order to investigate the relationship between different standard deviations in capacitance measurements and DNA-probe film structures at the nano-scale.

\subsection{DNA capacitance detection with 6-mercapto-1-hexanol layer}

DNA hybridization detection by using SH-terminated probes directly immobilized onto gold of a chip electrode and then posttreated with 6-mercapto-1-hexanol is summarized in Fig. 6. Acquisitions on different chip spots present similar results. In that case, standard deviations for both probe and target DNA are not small, as indicated in the figure. In case of DNA target detection, data acquired in $10 \mathrm{~min}$ on the same spot presents a signal variation up to $19.7 \%$. The capacitance signal on target is in the range $1.10-1.37 \mathrm{nF}$.

\subsection{DNA capacitance detection with Lipa-DEA layer}

DNA hybridization detection by using the SH-terminated oligos co-immobilized with Lipa-DEA onto gold of the chip electrodes is summarized in Fig. 7. Acquisitions on different 
chip spots present similar results. In that case, standard deviations for both probe and target DNA are much smaller than in the previous case, as indicated in the figure. In case of DNA target detection, the data acquired in $10 \mathrm{~min}$ on the same spot presents a signal variation of $1.1 \%$. The signal on target is in the

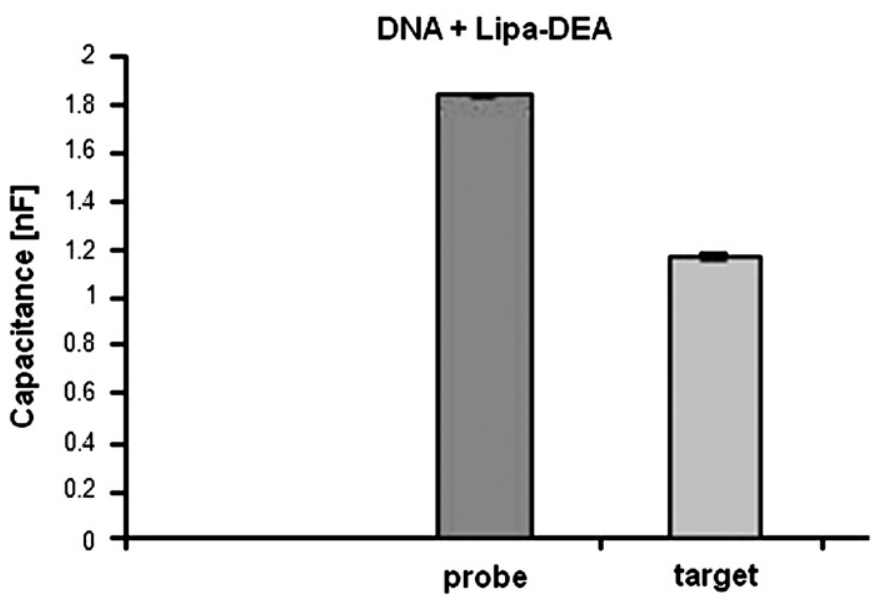

Fig. 7. DNA detection by using Lipa-DEA as blocking agent.

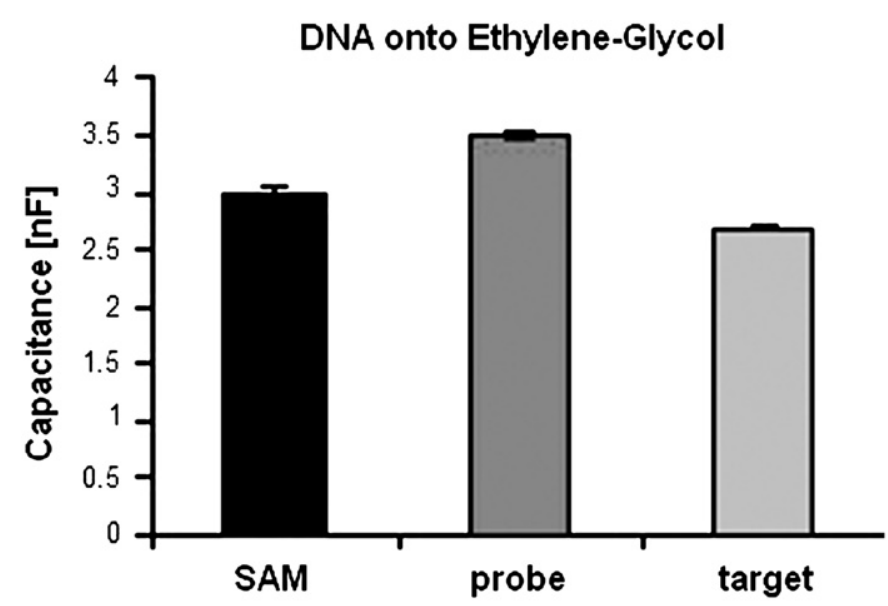

Fig. 8. DNA detection by using DNA probes immobilized onto ethylene-glycol precursor film. range $1.83-1.85 \mathrm{nF}$. Fig. 5 shows the hybridization phase as registered by SPR.

\subsection{DNA capacitance detection with ethylene-glycol probes layer}

DNA hybridization detection by using NH-terminated probes immobilized onto ethylene-glycol monolayers previously formed on chip gold of the biochip are summarized in Fig. 8. Acquisitions on different chip spots were similar. In that case, the data are related to capacitance acquired on ethylene-glycol SAM as well as on DNA probes and targets. Standard deviations are now smaller than in the two previous cases. In case of DNA target detection, data acquired in $10 \mathrm{~min}$ on the same spot presents a signal variation of only $0.6 \%$. The signal on target is in the range $4.78-$ $4.75 \mathrm{nF}$.

\subsection{AFM analysis on the probe surfaces}

AFM imaging is performed in order to verify the probes film structures at the nano-scale. Fig. 9(A) shows AFM image on a probe surface realized onto a template striped gold (TSG) by using SH-terminated ssDNA as probe and 6-mercapto-1-hexanol as blocking agent. The figure clearly shows evident grooves and distortions on the surface. Fig. 9(B) shows that those groves are actually very deep. Five AFM-tip deflections larger than $10 \mathrm{~nm}$ are clearly evident in the profile. Very large grooves are, instead, absent from the image showed in Fig. 10. This image is acquired on a surface prepared with SH-terminated ssDNA as probe and Lipa-DEA as blocking agent. This image presents a surface with small clots where very large grooves seem to be totally absent. The features of Fig. 10(A) are much more closely packed with respect to features showed in Fig. 9. However, profile analysis reveals some groves. Although poorly presented in such films, these groves may by deep. Fig. 10(B) shows an evident groove with deepness larger than $3 \mathrm{~nm}$ with respect to the average line profile. This means that groves crossing the whole probes film are possible in case of ssDNA immobilized with Lipa-DEA molecules, too. On the other hand, smoother features are registered by Fig. 11, which shows a surface where neither grooves nor clots are present. In this case, the surface average corrugation is smaller than in the two previous cases. Only AFM-tip deflections smaller than $1 \mathrm{~nm}$ are present in the profile of Fig. 11(B). This proves that the films obtained by using ethylene-glycol alkanethiols result in much more densely packed mono-layers in which average corrugation is very small and deep groves are totally absent.
A

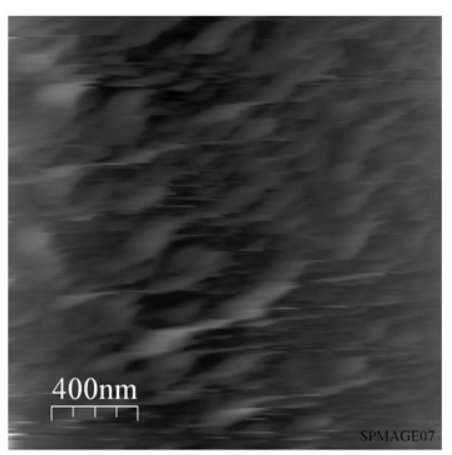

B

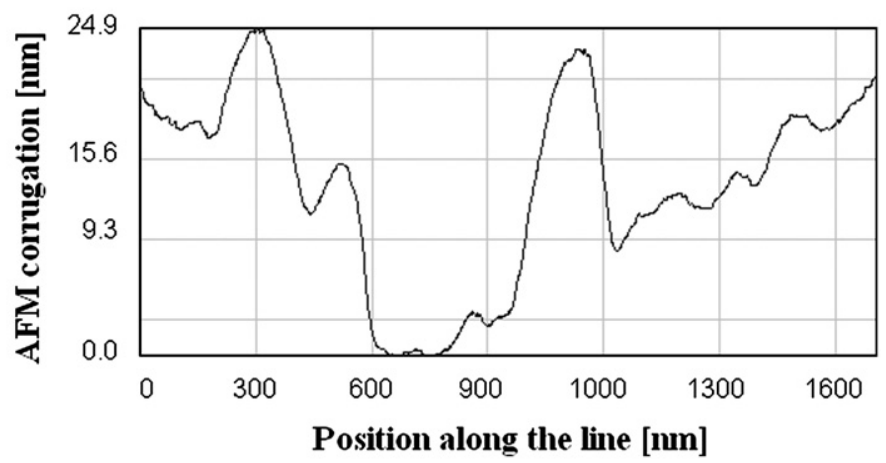

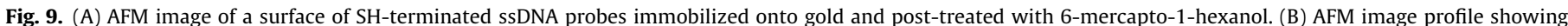
large tip deflections up to $15 \mathrm{~nm}$ on such a probes surface. 
A

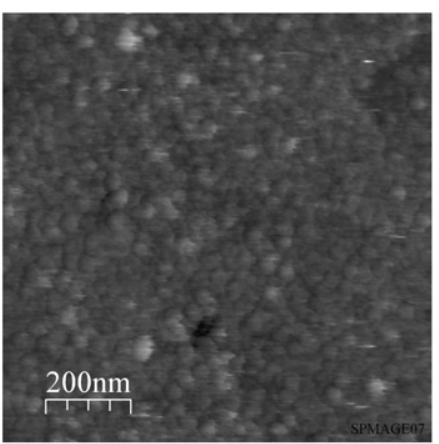

B

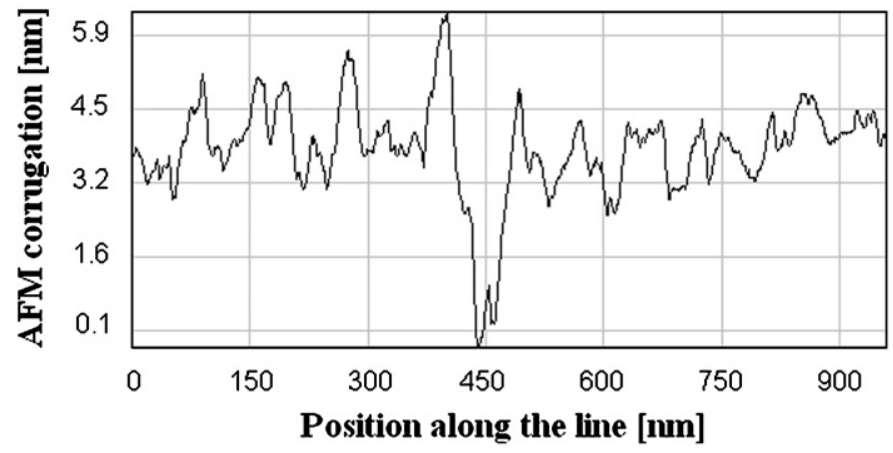

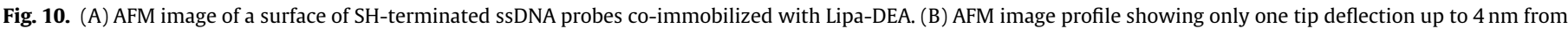
average corrugation on such a probes surface.

A

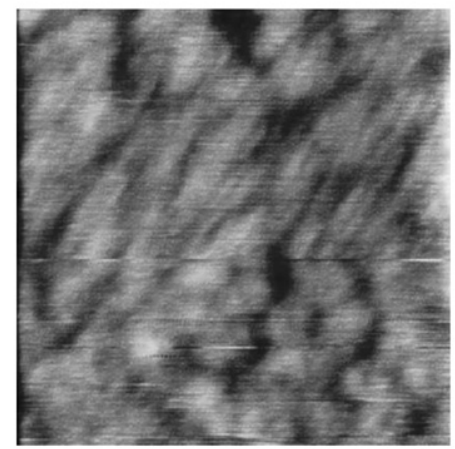

$B$

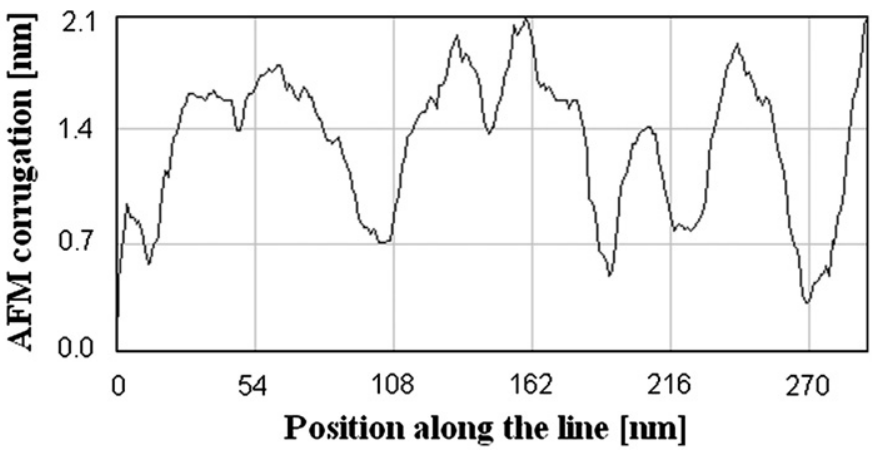

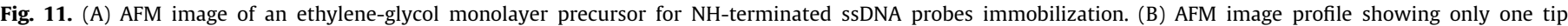
deflections below $1 \mathrm{~nm}$ are registered on such a probes surface.

\section{Discussion}

Figs. 6-8 compare the detection behaviors of DNA probe surfaces obtained with the different immobilization techniques showed in Fig. 3. A decreasing capacitance is observed in all three DNA target detections presented in these graphs. Similar capacitance decrease has also been observed after antibodies immobilization onto alkanethiols without ethylene-glycol function [24]. This decreasing capacitance upon target hybridization has been related to ions removal due to non-hydrophilic target molecules onto the probe surfaces $[2,4]$. The capacitance decreases because a conducting aqueous solution is being replaced by a hydrophobic target. Capacitance changes because additional molecules fill up unoccupied sites displacing some of the diffuse layers further out into solution [5]. A capacitance increase is instead showed by Fig. 8 after DNA probes immobilization onto the ethylene-glycol monolayer. Similar increases were also observed after antibody immobilization onto ethylene-glycol monolayers [24]. Such capacitance increases were associated to a net charge contribution of the probes molecules onto a hydrophilic surface [23]. By comparing the three figures, it is clearly evident that signal errors in Fig. 6 are larger than those in Figs. 7 and 8. The signal errors are larger on target DNA and on DNA probes, too. This is due to large time instability registered on surfaces made with 6-mercapto-1-hexanol. In our measurements, we registered capacitance variations in time from 1.10 up to $1.37 \mathrm{nF}$. Similar time instability was registered in case of probes immobilized without blocking agents [13]. There time instabilities are related to ion pathways that are present into probes layers [17], as schematically shown by Figs. 3(A) and (B). Such pathways were identified in large grooves showed by AFM images acquired on not well-packed films [23]. Similarly, Fig. 9 shows evident grooves in the AFM image acquired on probe surfaces with 6-mercapto-1-hexanol. DNA detection by using Lipa-DEA as blocking agent or ethylene-glycol anchoring precursors is more precise resulting in quite stable capacitance signals after DNA targets hybridization. Data of Fig. 7 present only a small $1.1 \%$ of capacitance change within $10 \mathrm{~min}$ of acquisition on probes film prepared treated with Lipa-DEA. Improved surface stability in case of Lipa-DEA used as blocking agent is also confirmed by SPR investigation, as reported in Fig. 5. In cases of Lipa-DEA, the AFM images present very few groves, as confirmed by Fig. 10(A) but sometime these groves are deep, as confirmed by Fig. 10(B). This fit quite well with a much more stable-in-time capacitance measurements obtained by immobilizing the ssDNA probes with Lipa-DEA as blocking agent. Fig. 8 presents the smaller $0.6 \%$ variation of capacitance in case of ethylene-glycol. The result fit quite well with the AFM analysis on same films which shows absolutely flat regions in Fig. 11(A), and absence of deep groves in the image profiles, as confirmed by Fig. 11(B). The AFM-tip deflection-amplitudes diminish in comparing Figs. 9(B), 10(B), and 11(B) which is a clear evidence of an increasing film ordering by passing from mercapto-hexanol to Lipa-DEA as blocking agents, and to ethylene-glycol as precursor films. More packed structures obtained by using ethylene-glycol precursors or Lipa-DEA blocking agent are also evident by comparing the average corrugation estimated in AFM images. It ranges from $4.2 \mathrm{~nm}$ in case of probe films with 6-mercapto-1-hexanol to 
1.9 and $0.4 \mathrm{~nm}$ for films with Lipa-DEA and ethylene-glycol, respectively. Presence of grooves in AFM images is so relevant because direct electron transfer between solution ions and gold electrode happens by through deep grooves. The presence of groves reported by the AFM image in Fig. 9 is a direct proof that gold electrode surface is accessible to solution ions in the films prepared by using 6-mercapto-1-hexanol. The reason of an improved performance in DNA capacitance detection by using Lipa-DEA is related to the presence of two sulfur and two hydroxyl groups in the molecule. The two sulfur groups assure a more stable anchoring of blocking agents onto gold surface while the two hydroxyl groups assure an improved hydrophilic character of the surface. A hydrophilic surface is assured even well by ethylene-glycol chains. Retained water is important to prevent ions penetration into probes film.

Presence of water strongly coordinated by ethylene-glycol chains was envisaged by $a b$ initio calculations and it has been confirmed by infrared spectroscopy and QCM [23]. The strong role played by water in film behavior is also confirmed by the improvement of capacitance stability after water conditioning and by a probe surface behavior close to that of an ideal insulator [23]. The presence of this stable water layer highly coordinated by ethylene-glycol chains is also indirectly showed by the blurred image in Fig. 11(A).

\section{Conclusions}

The originality of this paper is in the identification of surface functionalizations that drastically reduce time drift of interface impedance and, therefore, enhances DNA detection via capacitance measurements. We described new protocols to immobilize single strand DNA probe molecules onto capacitive biochip by means of $\mathrm{COOH}$-terminated ethylene-glycol alkanethiols used as anchoring precursors or by means of Lipa-DEA used as blocking agents. Capacitance measurements were analyzed in terms of signal errors and data ranges. It has been proved that sensing surfaces prepared with ethylene-glycol alkanethiols or with Lipa-DEA di-thiols present the best performances. Detection with ethylene-glycol precursors presents the smaller standard deviations while that with Lipa-DEA presents the higher detection signals. In both the cases, the detection is more stable-in-time than that with mercapto-hexanol which is usually considered as blocking agent. The improved stability of these new surfaces was explained in terms of ions pathways absence into the probes sensing surfaces. This explanation was demonstrated by AFM imaging and profiles analysis. DNA probe surfaces obtained with 6-mercapto-1-hexanol present very deep groves as such ion pathways. AFM analysis on ethylene-glycol films and DNA probes coimmobilized with Lipa-DEA does not reveal very deep grooves and shows highly packed structures with smaller surface corrugations. In conclusion, we have demonstrated that the new proposed probe functionalizations are highly suitable and reliable for applications in label-free capacitance detection with DNA biochip.

\section{Acknowledgments}

Biosensors and Bioelectronics 24 (2009) 3425-3429Frank K. Gürkaynak and Carlotta Guiducci are acknowledged for their support on biochip micro-fabrication. Claudio Stagni and Guido Zamagni are acknowledged for developing lab set-ups implementing CBCM and FTCM methods. The research has been financially supported by the European Project FP6 DINAMICS.

\section{References}

[1] Jiun H. Lin, Pharmacokinetics and pharmacodynamic viariability: a daunting challenge in drug therapy, Current Drug Metabolism 8 (2007) 109-136.

[2] Vladimir M. Mirsky, Michael Riepl, Otto S. Wolfbeis, Capacitive monitoring of protein immobilization and antigen-antibody reactions on monomolecular alkylthiol films on gold electrodes, Biosensors and Bioelectronics 12 (1997) 977-989

[3] Michael Riepl, Vladimir M. Mirsky, Ivan Novotny, Vladimir Tvarozek, Vlastimil Rehacek, Otto S. Wolfbeis, Optimization of capacitive affinity sensors: drift suppression and signal amplification, Analytica Chimica Acta 392 (1999) 77-84.

[4] Christine Berggren, Per Stalhandske, Jan Brundell, Gillis Johnansson, A feasibility study of a capacitive biosensor for direct detection of DNA hybridization, Electroanalysis 11 (1999) 156-160.

[5] Christine Berggren, Bjarni Bjarnason, Gillis Johnansson, An immunological Inerleukine-6 capacitive biosensor using perturbation with a potentiostatic step, Analytica Chimica Acta 387 (1998) 235-244.

[6] Philippe Corbisier, Daniel van der Lelie, Brigitte Borremans, Ann Provoost, Victor de Lorenzo, Nigel L. Brown, Jonathan R. Lloyd, Jonathan L. Hobman, Elisabeth Csoregi, Gillis Johansson, Bo Mattiasson, Whole cell- and proteinbased biosensors for the detection of bioavailable heavy metals in environmental samples, Analytica Chimica Acta 387 (1999) 235-244.

[7] C. Guiducci, C. Stagni, G. Zuccheri, A. Bogliolo, L. Benini, B. Samorì, B. Riccò, DNA detection by integrable electronics, Biosensors and Bioelectronics 19 (2004) 781-787.

[8] Anupama Balasubramanian, Bharat Bhuva, Ray Mernaugh, Frederick R. Haselton, Si-based sensor for virus detection, IEEE Sensors Journal 1530437 (2005) 1-5.

[9] A. Macanovic, C. Marquette, C. Polychronakos, M.F. Lawrence, Impedancebased detection of DNA sequences using a silicon transducer with PNA as the probe layer, Nucleic Acids Research 32 (2004) 1-7.

[10] Ibolya Bontidean, Josefin Ahlqvist, Ashok Mulchandani, Wilfred Chen, Weon Bae, Rajesh K. Mehra, Alessia Mortari, Elisabeth Csoregi, Novel synthetic phytochelatin-based capacitive biosensor for heavy metal ion detection, Biosensors and Bioelectronics 18 (2003) 547-553.

[11] C.A. Betty, R. Lal, D.K. Sharma, J.V. Yakhmi, J.P. Mittal, Macroporous silicon based capacitive affinity sensor-fabrication and electrochemical studies, Sensors and Actuators B 97 (2004) 334-343.

[12] Warakorn Limbut, Proespichaya Kanatharana, Bo Mattiasson, Punnee Asawatreratanakul, Panote Thavarungkul, A comparative study of capacitive immunosensors based on self-assembled monolayers formed from thiourea, thioctic acid, and 3-mercaptopropionic acid, Biosensors and Bioelectronics 22 (2006) 233-240.

[13] Claudio Stagni, Carlotta Guiducci, Luca Benini, Bruno Riccò, Sandro Carrara, Bruno Samorí, Christian Paulus, Meinrad Schienle, Marcin Augustyniak, Roland Thewes, CMOS DNA sensor array with integrated A/D conversion based on label-free capacitance measurement, IEEE Journal of Solid-State Circuits 41 (2006) 2956-2964.

[14] Claudio Stagni, Carlotta Guiducci, Luca Benini, Bruno Riccò, Sandro Carrara, Christian Paulus, Meinrad Schienle, Roland Thewes, A fully electronic labelfree DNA sensor chip, IEEE Sensors Journal 7 (2007) 577-585.

[15] Magdalena Gabig-Ciminska, Developing nucleic acid-based electrical detection systems, Microbial Cell Factories 5 (2006) 1-8.

[16] J.S. Daniels, N. Pourmand, Label-free impedance biosensors: opportunities and challenges, Electroanalysis (19) (2007) 1239-1257.

[17] Sandro Carrara, Frank K. Gürkaynak, Carlotta Guiducci, Claudio Stagni, Luca Benini, Yusuf Leblebici, Bruno Samorì, Giovanni De Micheli, Interface layering phenomena in capacitance detection of DNA with biochips, Sensors and Transducers Journal 76 (2007) 969-977.

[18] Inger Vikholm-Lundin, Reetta Piskonen, Binary monolayers of singlestranded oligonucleotides and blocking agent for hybridization, Sensors and Actuators B 134 (2008) 189-192.

[19] Inger Vikholm-Lundin, Sanna Auer, Tony Munter, Heidi Fiegl, Sophia Apostolidou, Hybridization of binary monolayers of single stranded oligonucleotides and short blocking molecules, Surface Science 603 (2009) 620-624.

[20] Emanuele Ostumi Lin Yan, George M. Whitesides, The interaction of proteins and cells with self-assembled monolayers of alkanethiolates on gold and silver, Colloids and Surface B: Biointerfaces 15 (1999) 3-30.

[21] Joydeep Lahiri, Lyle Isaacs, Joe Tien George M. Whitesides, A strategy for the generation of surfaces presenting ligands for studies of binding based on an active ester as a common reactive intermediate: a surface Plasmon resonance study, Analytical Chemistry 71 (1999) 777-790.

[22] K. Tappura, I. Vikholm-Lundin, W.M. Albers, Lipoate-based imprinted selfassembled molecular thin films for biosensor applications, Biosensors and Bioelectronics 22 (2007) 912-919.

[23] Sandro Carrara, Vijayender Kumar Bhalla, Claudio Stagni, Luca Benini, Anna Ferretti, Andrea Cavallini, Bruno Riccò, Bruno Samorì, New insights for using self-assembly materials to improve the detection stability in label-free DNA-chip and immunosensors, Biosensors and Bioelectronics 24 (2009) 3425-3429.

[24] Sandro Carrara, Vijayender Kumar Bhalla, Claudio Stagni, Luca Benini, Bruno Riccò, Bruno Samorì, Improving probe immobilization for label-free capacitive detection of DNA hybridization on microfabricated gold electrodes, Sensors and Transducers 88 (2008) 31-39. 